\title{
Phylogenetic analysis reveals gene conversions in multigene families of rhizobia
}

\author{
J.E. Hernandez-Salmeron and G. Santoyo \\ Laboratorio de Recombinacion y Diversidad Genomica, \\ Instituto de Investigaciones Quimico Biologicas, \\ Universidad Michoacana de San Nicolas de Hidalgo, Morelia, \\ Michoacan, Mexico \\ Corresponding author: G. Santoyo \\ E-mail: gsantoyo@umich.mx / gustavo_santoyo@yahoo.com
}

Genet. Mol. Res. 10 (3): 1383-1392 (2011)

Received October 21, 2010

Accepted January 16, 2011

Published July 12, 2011

DOI 10.4238/vol10-3gmr1118

\begin{abstract}
Gene families are an important and intrinsic trait of rhizobial species. These gene copies can participate in non-reciprocal recombination events, also called gene conversions. Gene conversion has diverse roles, but it is usually implicated in the evolution of multigene families. Here, we searched for gene conversions in multigene families of six representative rhizobial genomes. We identified 11 gene families with different numbers of copies, genome location and function in CFN42 and CIAT652 strains of Rhizobium etli, Rhizobium sp NGR234, Mesorhizobium loti MAFF303099, Sinorhizobium meliloti 1021, and Bradyrhizobium japonicum USDA110. Gene conversions were detected by phylogenetic inference in the nifD and nifK gene families in $R$. etli. Sequence analysis confirmed multiple gene conversions in these two gene families. We suggest that gene conversion events have an important role in homogenizing multigene families in rhizobia.
\end{abstract}

Key words: Rhizobia; Gene conversion; Concerted evolution; Multigene families 


\section{INTRODUCTION}

Bacteria usually contain multigene families as an intrinsic trait of their genomes (Achaz et al., 2002; Treangen et al., 2009). Some of them exhibit extraordinary similarity between all its members, which suggests a recent origin after duplication, or a homogenizing mechanism called gene conversion (Santoyo and Romero, 2005). Gene conversion has been widely documented in diverse multigene families, including the rRNA genes in Escherichia coli (Liao, 2000), tuf in Salmonella typhimurium (Abdulkarim and Hughes, 1996) and Vibrio cholerae (Lathe III and Bork, 2001), pilE in Neisseria gonorrhoeae and N. meningitidis (Haas and Meyer, 1986; Kline et al., 2003), and hop in Chlamydia pneumoniae and Helicobacter pylori (Jordan et al., 2001), among others (Santoyo and Romero, 2005). Gene conversion also plays essential roles in antigenic variation, an important mechanism in bacterial pathogens to evade the host immune system, as well as other positive and detrimental roles (Deitsch et al., 1997).

In rhizobial genomes, gene conversion has been studied only in the nifH family of Rhizobium etli, which codes for the enzyme nitrogenase (Rodriguez and Romero, 1998; Santoyo et al., 2005). However, it is known that $R$. etli and other rhizobial genera contain a huge amount of reiterated DNA sequences, including multigene families, which can be targets for gene conversion events (Gonzalez et al., 2006; Orozco-Mosqueda et al., 2009). Genome analysis of $R$. etti shows the presence of 133 families of identical repeats, while Sinorhizobium meliloti displays 24 long sequence repeats (Gonzalez et al., 2006; Orozco-Mosqueda et al., 2009). Other rhizobia such as Mesorhizobium loti and Bradyrhizobium japonicum have been completely sequenced, but the number of gene families is not mentioned (Kaneko et al., 2000, 2002). Although some rhizobia have a huge amount of identical repeats, not all of them belong to gene coding families.

Gene conversion is experimentally difficult to study in bacteria, due to the problem of recovering all products of a gene conversion event (Liao, 2000). Therefore, phylogenetic inference has been useful to study this mechanism in different gene families in bacteria. For example, multiple independent gene conversion events were detected in outer membrane proteins of $H$. pylori and C. pneumoniae (Jordan et al., 2001). In another study, gene conversions were also detected by phylogenetic evidence in the tuf genes of different bacteria (Lathe III and Bork, 2001).

In this study, gene conversion was surveyed in 11 multigene families of $R$. etli, strains CFN42 and CIAT652, by phylogenetic analysis. Gene conversions were also searched in orthologs of the multigene families in other complete rhizobial genomes, such as Rhizobium sp NGR234 (Schmeisser et al., 2009), M. loti MAFF303099 (Kaneko et al., 2000), S. meliloti 1021 (Galibert et al., 2001), and B. japonicum USDA110 (Kaneko et al., 2002).

\section{MATERIAL AND METHODS}

\section{Identification of multigene families}

Members of multigene families were identified by literature search in PUBMED (http:// www.ncbi.nlm.nih.gov/pubmed) of the National Center for Biotechnology Information (NCBI). Eleven multigene families of the R. etli CFN42 and CIAT652 strains were chosen and analyzed in this study because of their biological significance. It is worth noting that other long DNA repeats are present in the R. etli genome; however, our study focused on gene coding families. The nucleotide sequence of each copy was obtained from GenBank (NCBI) with the following GenID 
numbers: $R$. etli CFN42, nifH (1005068, 1005033, 1004995), nifD (1005069, 1004996), nifK (1005079, 1004997), nodD (6402815, 6402862, 6402864), ccm (3896046, 3896045, 3896047), adhC (3895921, 3892269), tuf (3892582, 3892567), fix (1005327, 1005055, 1005054), groEL (3894519, 3891062), dnaJ (3890921, 3890921, 3894442), and purU (6400149, 6398142); $R$. etli CIAT652, nifH (6402850, 6402804, 6402760), nifD (6402759, 6402805, 6402849), nifK (6402758, 6402848), nodD (6402815, 6402862, 6402864), fix (6402772, 6402773, 6402774), and groEL (6403417, 6399413); Rhizobium sp NGR234, nifH (962478, 962500), nifD (962502, 962482), nifK (962485, 962471), fix (962188, 962189), groES (7788251, 7791635), groEL (7792170, 7788321, 7788250, 7791634), and dnaJ (7792773, 7791032, 7791742); R. leguminosarum, fix (4403906, 4403966), groEL (4402951, 4398962, 4401984, 4398208), etfA (4401546, 4398246), etfB (4401547, 4398247), and hemA (4398963, 4402319); M. loti, nifA (1229107, 1229090, 1229044), герA (1224477, 1231331), герB (1224476, 1231330), герC (1224475, 1231329), groES (1231535, 1230998, 1226411, 1226287, 1229067), groEL (1231471, 1230997, $1226412,1226286,1229066)$, and dnaJ (1228214, 1230424); B. japonicum, groES (1053428, 1055491, 1048455, 1054913, 1051464), groEL (1053427, 1052432, 1055489, 1048307, 1051467, 1051462, 1047245), rpoN (1054956, 1054195), dnaJ (1052529, 1052410, 1049300, 1048922), and fix (1055510, 1054820, 1054853), and S. meliloti nod (1235512, 1235224, 1235494), fix (1235483, 1235482), fixO3 (1235141, 1235447, 1235700), groES (1235290, $1235216,1232434)$, and groEL $(1235289,1235215,1237337,1232840,1232433)$.

Orthologs of 11 multigene families were also searched in other rhizobia by similarity blast genome research (http://blast.ncbi.nlm.nih.gov/Blast.cgi). Genomes analyzed included Rhizobium sp NGR234 (NC_012587.1), R. leguminosarum (NC_008380.1), B. japonicum (NC_004463.1), S. meliloti (NC_003047.1), and M. loti (NC_002678.2).

\section{Phylogenetic analysis}

A multiple sequence alignment was generated with ClustalX, and the phylogenetic analysis of the multigene families was carried out with the MEGA 4.0 program (Tamura et al., 2007). To obtain a confidence value for the aligned sequence dataset, a bootstrap analysis of 1000 replications was done. Phylogenetic trees were constructed by the neighbor-joining method based on Kimura's two-parameter distance (Kimura, 1980). Other methods were also employed, but irrespective of that, the overall tree topologies were similar in all cases. Alignment analysis of the gene sequences was done with the help of the ClustalW program (http:// www.ebi.ac.uk/Tools/clustalw2/).

\section{RESULTS}

\section{Identification of multigene families in rhizobia}

Rhizobial genomes are an interesting model to study recombination and genome dynamics mechanisms, due to the wide presence of multigene families. In some cases, these multiple gene copies are located on different replicons, either chromosome or plasmids. Recombination between these inter-replicon copies may lead to diverse genome rearrangements (Orozco-Mosqueda et al., 2009).

Here, we identified and analyzed 11 multigene families in two R. etli strains (CFN42 
and CIAT652). Some of these gene families were also found in the genome of other rhizobia. For example, the chaperonin groEL gene family was found to be reiterated in all the rhizobial genomes analyzed in this study. The number of gene copies varied: 4 copies are present in $R$. etli, Rhizobium sp NGR234 and R. leguminosarum, 5 in S. meliloti and M. loti, and 7 in B. japonicum. Other gene families, including nifH, are present in two or three copies in the Rhizobium genera, but not in others. Table 1 shows the list and copy number of the gene families analyzed here for gene conversions. It is interesting that diverse gene copies are distributed in different replicons, which can be targets for recombination, thereby generating genome rearrangements such as deletions, inversions and amplifications. This is an interesting subject for further studies.

Table 1. Multigene families in the order Rhizobiales.

\begin{tabular}{|c|c|c|c|c|}
\hline Genome & Multigene family & Function & Number of copies & Genomic localization \\
\hline \multirow[t]{11}{*}{ Rhizobium etli CFN42 } & nifH & Nitrogenase reductase & 3 & Plasmid \\
\hline & nifD & Nitrogenase subunit $\alpha$ & 3 & Plasmid \\
\hline & $n i f K$ & Nitrogenase subunit $\beta$ & 2 & Plasmid \\
\hline & nodD & Transcriptional regulator & 3 & Plasmid \\
\hline & $\mathrm{ccm}$ & Cytochrome c & 3 & Chromosome/plasmid (3) \\
\hline & $a d h C$ & Formaldehyde dehydrogenase & 2 & Chromosome/plasmid \\
\hline & tuf & Elongation factor & 2 & Chromosome \\
\hline & fix & Electron transfer & 3 & Plasmid \\
\hline & groEL & Chaperonin & 4 & Chromosome (3)/plasmid \\
\hline & dnaJ & Chaperone & 3 & Chromosome \\
\hline & $\operatorname{pur} U$ & Purine biosynthesis & 2 & Chromosome \\
\hline \multirow[t]{7}{*}{ Rhizobium sp NGR234 } & nifH & Nitrogenase reductase & 2 & Plasmid \\
\hline & $n i f D$ & Nitrogenase subunit $\alpha$ & 2 & Plasmid \\
\hline & nifK & Nitrogenase subunit $\beta$ & 2 & Plasmid \\
\hline & fixA & Electron transport & 3 & Plasmid \\
\hline & groES & Chaperonin & 2 & Chromosome/plasmid \\
\hline & groEL & Chaperonin & 4 & Chromosome (2)/plasmid (2) \\
\hline & dnaJ & Chaperone & 3 & Chromosome \\
\hline \multirow[t]{5}{*}{ Rhizobium leguminosarum } & $f i x$ & Electron transport & 2 & Plasmid \\
\hline & groEL & Chaperonin & 4 & Chromosome (2)/plasmid (2) \\
\hline & etfA & Electron acceptor & 2 & Chromosome/plasmid \\
\hline & etfB & Electron acceptor & 2 & Chromosome/plasmid \\
\hline & hemA & Coenzyme & 2 & Chromosome/plasmid \\
\hline \multirow[t]{7}{*}{ Mesorhizobium loti } & nifA & Regulatory protein & 3 & Chromosome \\
\hline & repA & Replication & 2 & Plasmid \\
\hline & rep $B$ & Replication & 2 & Plasmid \\
\hline & repC & Replication & 2 & Plasmid \\
\hline & groES & Co-chaperonin & 5 & Chromosome (4)/plasmid \\
\hline & groEL & Chaperonin & 5 & Chromosome (4)/plasmid \\
\hline & dnaJ & Chaperone & 2 & Chromosome \\
\hline \multirow[t]{5}{*}{ Bradyrhizobium japonicum } & groES & Co-chaperonin & 5 & Chromosome \\
\hline & groEL & Chaperonin & 7 & Chromosome \\
\hline & rpoN & Sigma factor & 2 & Chromosome \\
\hline & dnaJ & Chaperone & 4 & Chromosome \\
\hline & fix & Electron transport & 3 & Chromosome \\
\hline \multirow{5}{*}{ Sinorhizobium meliloti } & $\operatorname{nod} D$ & Regulation of nodulation & 3 & Plasmid \\
\hline & fix & Electron transport & 2 & Plasmid \\
\hline & fixO3 & Cytochrome c oxidase & 3 & Plasmid \\
\hline & groES & Chaperonin & 3 & Plasmid \\
\hline & groEL & Chaperonin & 5 & Chromosome (2)/plasmid (3) \\
\hline
\end{tabular}

\section{Phylogenetic analysis of multigene families}

The hypothesis states that orthologs present in two strains or very closely related species are more evolutionarily related than paralogous genes. This is because orthologs shared a 
more recent common ancestor at the time of strain divergence, while paralogous genes shared an ancestor at the time of duplication, previous to strain divergence (Jordan et al., 2001). When orthologous genes were not found as multigene families in some rhizobial genera, we were unable to prove the above hypothesis. However, it was really useful to have the complete genome sequence available for the two strains of $R$. etli (CFN42 and CIAT652).

Figure 1 shows the phylogeny of the groEL multigene family. This confirms the prediction that orthologous genes are more closely related than paralogs within the same genome, and therefore, evidence for gene conversions is lacking. In some cases, the groEL copies were grouped in the same clade for M. loti and B. japonicum, but these species are too divergent, and these orthologs were probably duplicated after species divergence. In addition, sequence analysis did not reveal gene conversions within this groEL family in any rhizobial analyzed here.

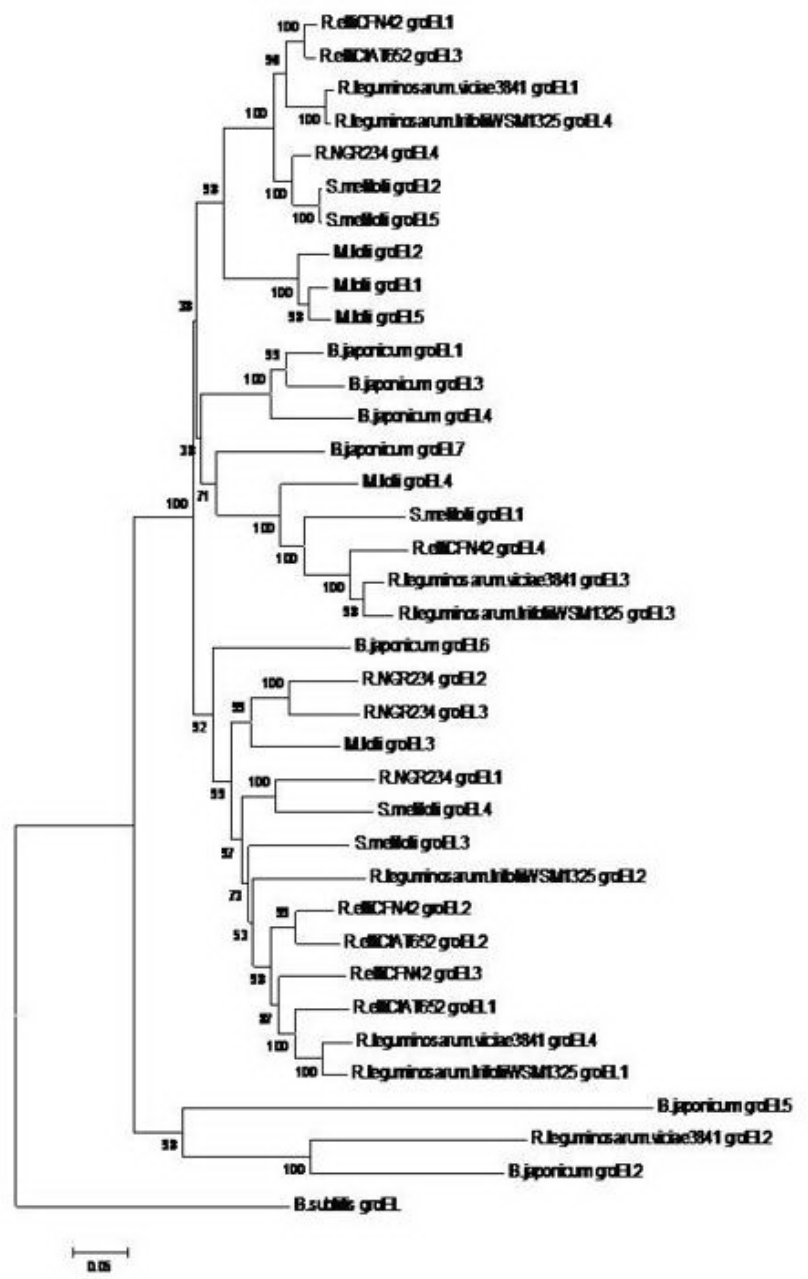

Figure 1. Phylogenetic tree of the groEL multigene family in diverse rhizobial genomes. A multiple sequence alignment was generated with ClustalX, and construction of the phylogeny was carried out by the neighbor-joining method of the MEGA 4.0 program. A bootstrap analysis of 1000 replications was done. 
Interestingly, the phylogenies of the nifD and nifK multigene families in $R$. etli strains clearly showed evidence of apparent gene conversions, because paralogs are more related than orthologs (Figures 2 and 3). The phylogenies of the rest of the multigene families in all the rhizobial genomes studied here did not show evidence of gene conversions (data not shown).

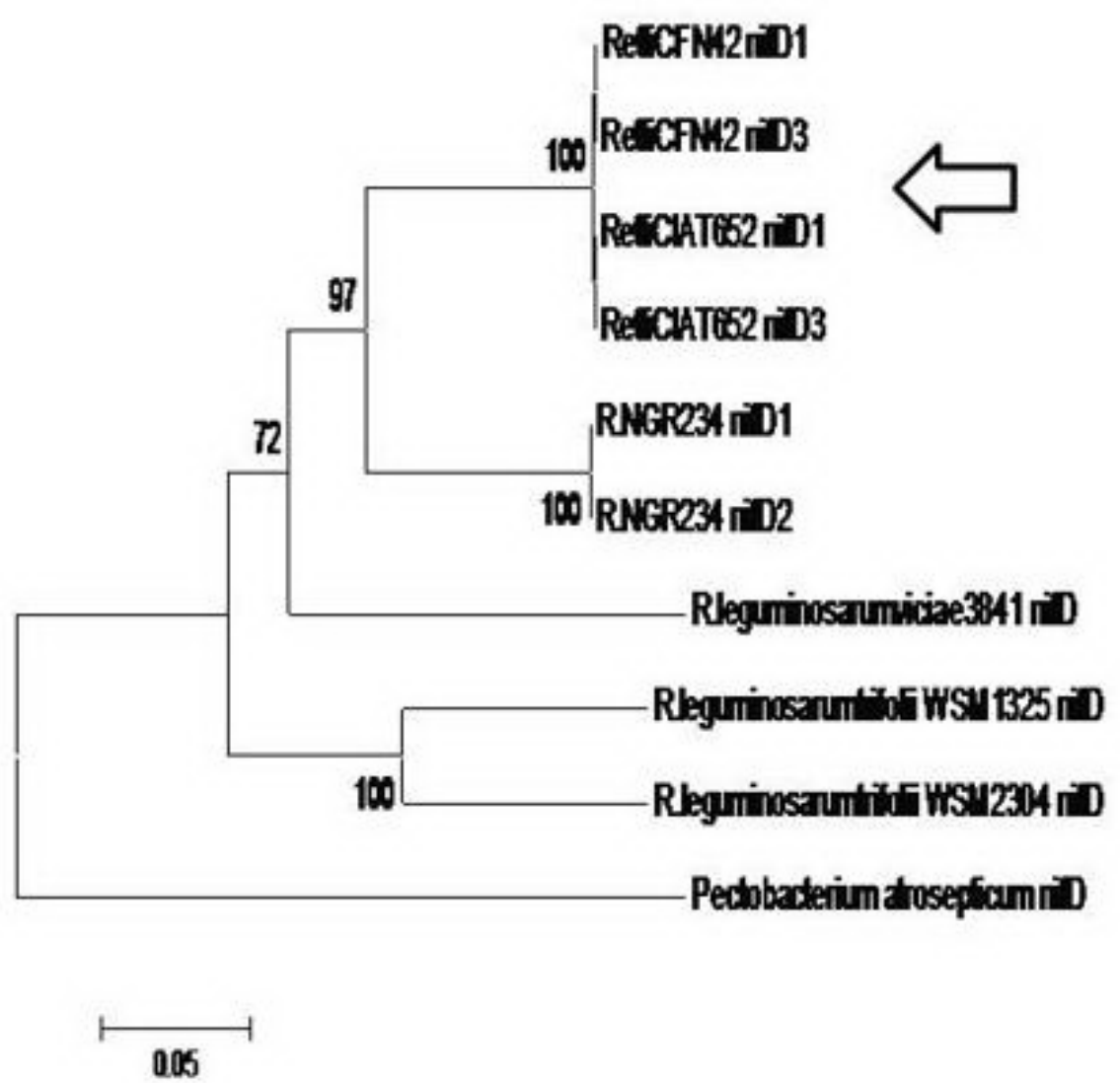

Figure 2. nifD multigene family phylogeny. Arrow indicates the clade of the probable gene conversions. See text for details.

\section{Sequence analysis of gene conversion events}

An alignment analysis of the nifD sequences reveals that the two paralogous gene copies in the R. etli CFN42 genome are completely identical, as well as the gene copies present within $R$. etli CIAT652. Nonetheless, when we compared the nifD sequences between strains, two sequence polymorphisms were detected (Figure 4). These nucleotide polymorphisms are indicative of probable gene conversion events. Sequence alignment of the nifK gene family in the two $R$. etli strains also reveals a more complex pattern of gene conversions, since we identified 13 polymorphisms between the orthologous copies (Figure 5). 


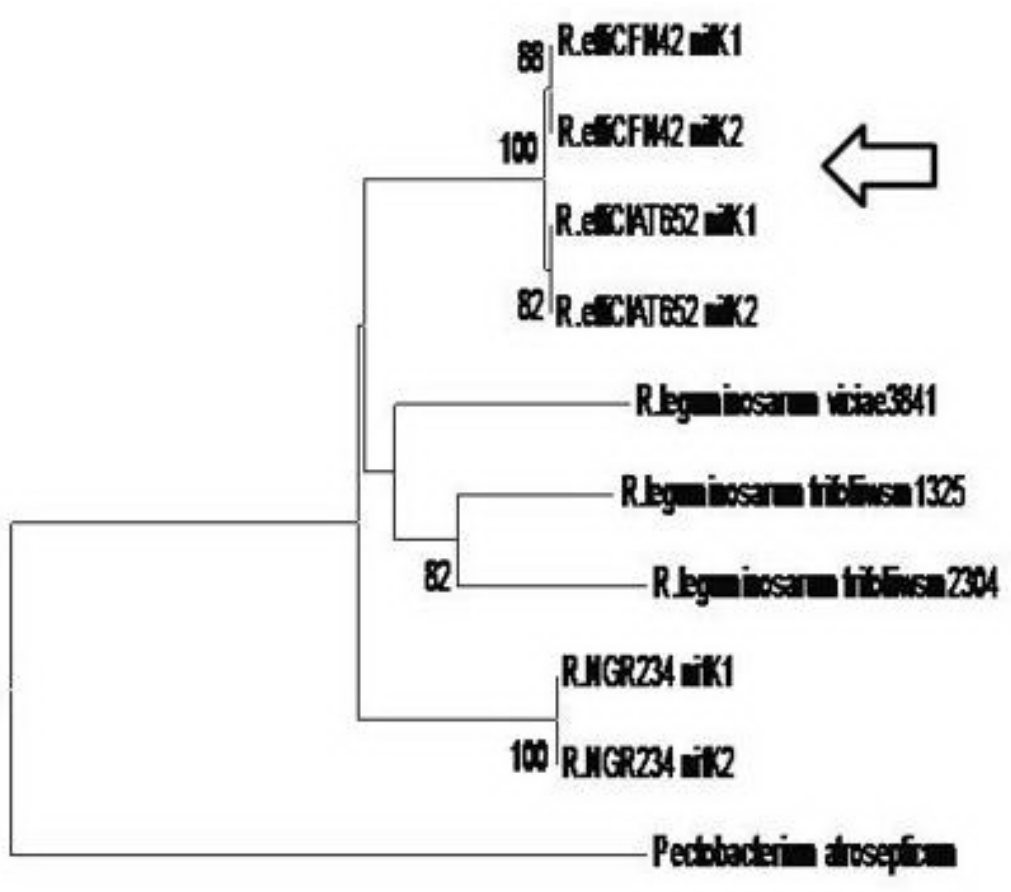

\section{5}

Figure 3. nifK multigene family phylogeny. Arrow indicates the clade of the probable gene conversions. See text for details.

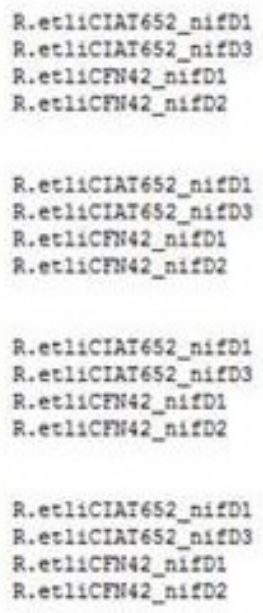

Figure 4. Sequence polymorphisms show evidence of gene conversion in the nifD multigene family (indicated by rectangles). 


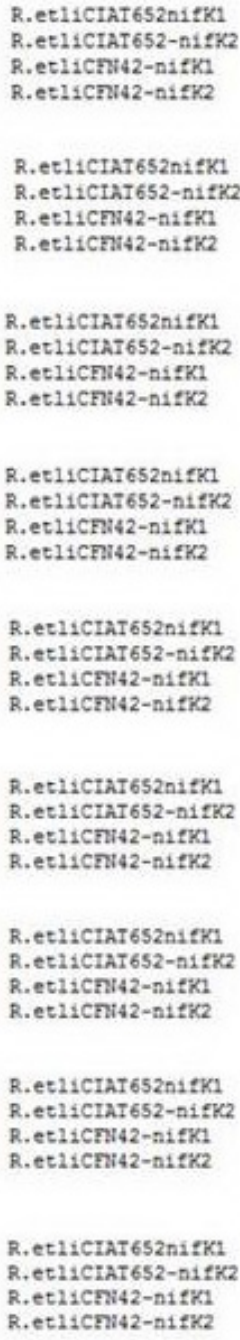

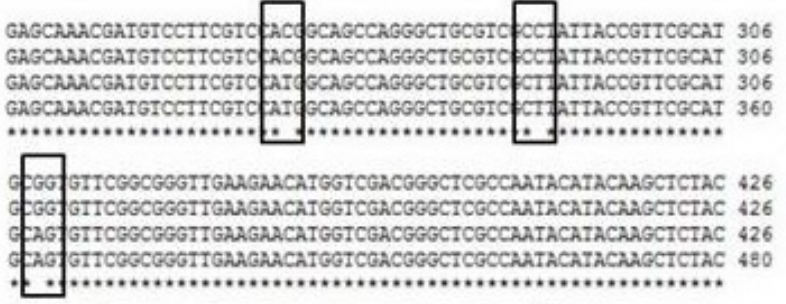

TTGAATGCCGAAGCGACCCTGICGCTGCAGCACTATAACACGCGC NGa GGCTGGAATAT 906

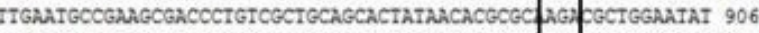
TTGAATGCCGAAGCGACCCTGTCGCTGCAGCACTATAACACGCGC PNA -GCTGGAATAT 906 TTGATGCCGAAGCACCCTGICGCTGCAGCACTATAACACGCGC MAS GCTGGAATAI 960

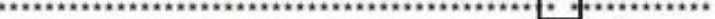

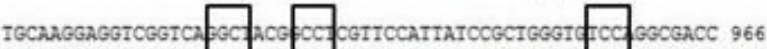
TGCAAGGAGGTCGGTCA GC ACG FCC CGITCCATTATCCGCT GGGICE EC GGCGACC 966 TGCAAGGAGGTCGGTCA PGI ACG CI CGITCCAITATCCGCTGGGI f ITC GGCGACC 966

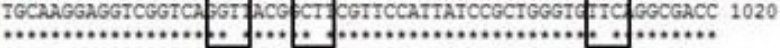

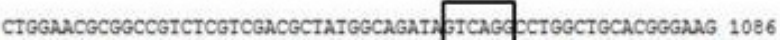
CTGGAACGCGGCCGTCTCGICGACGCTATGGCAGATA FTCAGC СТTGGTGCACGGGAAG 1086 CTGGAACGCGGCCGTCTCGICGACGCTATGGCAGAT: ECCAA ФCTGGCTGCACGGGAAG 1086 CTGGACGCGGCCGTCTCGICGACGCTATCGCAGAIA ECCAA ECTGGCTGCACGCGAAG 1140

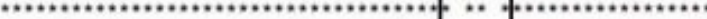

ACCGGCGGIGAGCCGACCCACTGCCITGCTACCARCGGCA GCD OCCTGGGAMGCCGAG 1206 ACCGGCGOTGAOCCGACCCACTOCCITGCTACCAACGGCA OCC AOCCTOGGAAGCCGAG 1206 ACCGGCGGIGAGCCGACCCACTGCCTIGCTACCARCGGCA GI FGCCTGGGMACCCGAG 1206 ACCGGCGGIGAGCCGACCCACTGCCTTGCTACCAACGGCA GICAGCCTGGGAAGCCGAG 1260

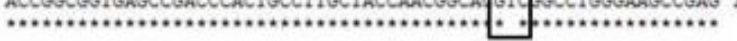

ATGAAGGCGTTGCTCGCATCCTCGCCCTICGG CaA ofatGCCCAGGTCTGGGCGGGCAAG 1266 ATGAAGGCGTTGCTCGCATCCTCGCCCTICGG CA gaTGCCCAGGTCTGGGCGGGCAAG 2266 ATGARGGCGITGCTCGCATCCTCGCCCITCGG PAR EATGCCCAGGTCTGGGCGGGCAAG 1266 ATGAAGCCGITGCTCGCATCCTCGCCCTICGG FAd GATGCCCAGGICTGGGCGGCCAAG 1320

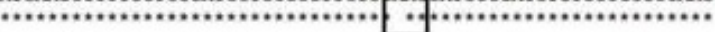

GACCTGIGGGCGITGCGCTCGCTGCTCTITACCG SCCAGTAG GACCTGTGGGCGITGCGCTCGCTGCTCTITACCG: GCCAGTAGA FCTTATGATCGGCANI 1326 GACCTGTGGGCGTIGCGCTCGCTGCTCTTTACCG: 3CCGGITGA CCTTATGATCGGCAMT 1326

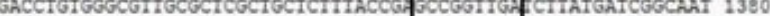

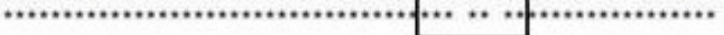

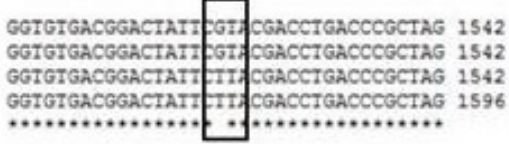

Figure 5. Multiple sequence polymorphisms show evidence of gene conversion in the nifK multigene family (indicated by rectangles).

\section{DISCUSSION}

An interesting outcome of a recombination event between identical or highly similar gene copies is the non-reciprocal transfer of genetic information, resulting in a homogenizing mechanism known as gene conversion. Gene conversion has been studied in diverse bacteria, where it plays an important role in the concerted evolution of multigene families (Santoyo and Romero, 2005).

This study provided phylogenetic evidence of gene conversions in two multigene 
families of rhizobia, which was also confirmed by sequence comparative analysis.

It has been proposed that transformation could be a homogenization mechanism in Hop genes in H. pylori (Alm et al., 1999). However, there is no evidence that R. etli is transformable. In fact, laboratory experiments have shown an extremely low efficiency in transforming it by diverse protocols and methods (Romero D, personal communication). Therefore, gene conversion appears to be the most probable homogenizing mechanism. Also, a study by Santoyo et al. (2005) showed experimentally that gene conversion can homogenize the nifH multigene family in $R$. etli. They also determined that the length of gene conversion events between the nifH copies is approximately 100 to $800 \mathrm{bp}$. For the nifD family, the two polymorphisms have a distance of $170 \mathrm{bp}$. However, for the nifK family, there are multiple gene conversions, with the longest distance between the first one and last one of $1254 \mathrm{bp}$. In this way, apparent gene conversions between the nifD and nifK paralogous gene copies are within this probable size range.

It is interesting to note that in bacterial pathogens, gene conversion could provide a selective advantage by homogenizing housekeeping genes or those implicated in survival in adverse environments (Deitsch et al., 1997; Liao, 2000). However, the biological significance of gene conversions in nif multigene families (other than evolving in a concerted way) is still unknown. These nif genes play no essential role in viability or survival, since Rhizobium species can live saprophytically. Nonetheless, nif genes are important during nodulation processes because they are expressed to fix nitrogen and promote plant health. The complete genome sequences of the other six $R$. etli strains from different geographical origins are currently being determined (González et al., 2010). Genomic comparison between the multigene families or long DNA repeats would reveal whether gene conversion is involved in not only homogenizing non-housekeeping functions but also other functions.

\section{ACKNOWLEDGMENTS}

Research supported by Coordinacion de la Investigacion Cientifica-UMSNH (Programa de Investigacion 2009/2010). J.E. Hernandez-Salmeron received a Master's Scholarship from CONACYT-Mexico (\#232463).

\section{REFERENCES}

Abdulkarim F and Hughes D (1996). Homologous recombination between the tuf genes of Salmonella typhimurium. J. Mol. Biol. 260: 506-522.

Achaz G, Rocha EP, Netter P and Coissac E (2002). Origin and fate of repeats in bacteria. Nucleic Acids Res. 30: $2987-$ 2994.

Alm RA, Ling LS, Moir DT, King BL, et al. (1999). Genomic-sequence comparison of two unrelated isolates of the human gastric pathogen Helicobacter pylori. Nature 397: 176-180.

Deitsch KW, Moxon ER and Wellems TE (1997). Shared themes of antigenic variation and virulence in bacterial, protozoal, and fungal infections. Microbiol. Mol. Biol. Rev. 61: 281-293.

Galibert F, Finan TM, Long SR, Puhler A, et al. (2001). The composite genome of the legume symbiont Sinorhizobium meliloti. Science 293: 668-672.

Gonzalez V, Santamaria RI, Bustos P, Hernandez-Gonzalez I, et al. (2006). The partitioned Rhizobium etli genome: genetic and metabolic redundancy in seven interacting replicons. Proc. Natl. Acad. Sci. U. S. A 103: 3834-3839.

Gonzalez V, Acosta JL, Santamaria RI, Bustos P, et al. (2010). Conserved symbiotic plasmid DNA sequences in the multireplicon pangenomic structure of Rhizobium etli. Appl. Environ. Microbiol. 76: 1604-1614.

Haas R and Meyer TF (1986). The repertoire of silent pilus genes in Neisseria gonorrhoeae: evidence for gene conversion. 
Cell 44: 107-115.

Jordan IK, Makarova KS, Wolf YI and Koonin EV (2001). Gene conversions in genes encoding outer-membrane proteins in H. pylori and C. pneumoniae. Trends Genet. 17: 7-10.

Kaneko T, Nakamura Y, Sato S, Asamizu E, et al. (2000). Complete genome structure of the nitrogen-fixing symbiotic bacterium Mesorhizobium loti. DNA Res. 7: 331-338.

Kaneko T, Nakamura Y, Sato S, Minamisawa K, et al. (2002). Complete genomic sequence of nitrogen-fixing symbiotic bacterium Bradyrhizobium japonicum USDA110. DNA Res. 9: 189-197.

Kimura M (1980). A simple method for estimating evolutionary rates of base substitutions through comparative studies of nucleotide sequences. J. Mol. Evol. 16: 111-120.

Kline KA, Sechman EV, Skaar EP and Seifert HS (2003). Recombination, repair and replication in the pathogenic Neisseriae: the 3 R's of molecular genetics of two human-specific bacterial pathogens. Mol. Microbiol. 50: 3-13.

Lathe WC III and Bork P (2001). Evolution of tuf genes: ancient duplication, differential loss and gene conversion. FEBS Lett. 502: 113-116.

Liao D (2000). Gene conversion drives within genic sequences: concerted evolution of ribosomal RNA genes in bacteria and archaea. J. Mol. Evol. 51: 305-317.

Orozco-Mosqueda MC, Altamirano-Hernandez J, Farias-Rodriguez R, Valencia-Cantero E, et al. (2009). Homologous recombination and dynamics of rhizobial genomes. Res. Microbiol. 160: 733-741.

Rodriguez C and Romero D (1998). Multiple recombination events maintain sequence identity among members of the nitrogenase multigene family in Rhizobium etli. Genetics 149: 785-794.

Santoyo G and Romero D (2005). Gene conversion and concerted evolution in bacterial genomes. FEMS Microbiol. Rev. 29: $169-183$.

Santoyo G, Martinez-Salazar JM, Rodriguez C and Romero D (2005). Gene conversion tracts associated with crossovers in Rhizobium etli. J. Bacteriol. 187: 4116-4126.

Schmeisser C, Liesegang H, Krysciak D, Bakkou N, et al. (2009). Rhizobium sp. strain NGR234 possesses a remarkable number of secretion systems. Appl. Environ. Microbiol. 75: 4035-4045.

Tamura K, Dudley J, Nei M and Kumar S (2007). MEGA4: molecular evolutionary genetics analysis (MEGA) software version 4.0. Mol. Biol. Evol. 24: 1596-1599.

Treangen TJ, Abraham AL, Touchon M and Rocha EP (2009). Genesis, effects and fates of repeats in prokaryotic genomes. FEMS Microbiol. Rev. 33: 539-571. 